\title{
Fast acceleration of "killer" electrons and energetic ions by inter- planetary shock stimulated ULF waves in the inner magnetosphere
}

\author{
ZONG QiuGang*, WANG YongFu, YUAN ChongJing, YANG Biao, WANG ChenRui \& \\ ZHANG XiangYun
}

Institute of Space Physics and Applied Technology, Peking University, Beijing 100871, China

Received August 30, 2010; accepted November 25, 2010

Energetic electrons and ions in the Van Allen radiation belt are the number one space weather threat. Understanding how these energetic particles are accelerated within the Van Allen radiation belt is one of the major challenges in space physics. This paper reviews the recent progress on the fast acceleration of "killer" electrons and energetic ions by ultralow frequency (ULF) waves stimulated by the interplanetary shock in the inner magnetosphere. Very low frequency (VLF) wave-particle interaction is considered to be one of the primary electron acceleration mechanisms because electron cyclotron resonances can easily occur in the VLF frequency range. Recently, using four Cluster spacecraft observations, we have found that, after interplanetary shocks impact the Earth's magnetosphere, energetic electrons in the radiation belt are accelerated almost immediately and continue to accelerate for a few hours. The time scale (a few days) for traditional acceleration mechanisms, based on VLF wave-particle interactions to accelerate electrons to relativistic energies, is too long to explain our observations. Furthermore, we have found that interplanetary shocks or solar wind pressure pulses, with even small dynamic pressure changes, can play a non-negligible role in radiation belt dynamics. Interplanetary shocks interaction with the Earth's magnetosphere manifests many fundamental space physics phenomena including energetic particle acceleration. The mechanism of fast acceleration of energetic electrons in the radiation belt responding to interplanetary shock impacts consists of three contributing parts: (1) the initial adiabatic acceleration due to strong shock-related magnetic field compression; (2) followed by the drift-resonant acceleration with poloidal ULF waves excited at different L-shells; and (3) particle acceleration due to the quickly damping electric fields associated with ULF waves. Particles end up with a net acceleration because they gain more energy in the first half of this cycle than they lose in the second. The results reported in this paper cast a new light on understanding the acceleration of energetic particles in the Earth's Van Allen radiation belt. The results of this study can likewise be applied to interplanetary shock interaction with other planets such as Mercury, Jupiter, Saturn, Uranus and Neptune, and other astrophysical objects with magnetic fields.

"killer" electrons, radiation belt, energetic particles acceleration, ULF wave, VLF wave, wave-particle interaction, resonance

Citation: Zong Q G, Wang Y F, Yuan C J, et al. Fast acceleration of "killer" electrons and energetic ions by inter-planetary shock stimulated ULF waves in the inner magnetosphere. Chinese Sci Bull, 2011, 56: 1188-1201, doi: 10.1007/s11434-010-4308-8

The Earth's geomagnetic field can be strongly affected by the impact of interplanetary shocks. During the early 1940s, the Earth's magnetic signal response to the passage of interplanetary shocks was recorded by ground-based magnetometer observations. Such magnetic field signals are known as "storm sudden commencement" (SSC) [1]. Ener-

*Corresponding author (email: qgzong@pku.edu.cn) getic particles in the magnetosphere can be accelerated by the hit of interplanetary shocks [2-5]. It has been recognized that energetic (tens of $\mathrm{keV}$ ) electron precipitation, which typically lasts 3 to $10 \mathrm{~min}$, would be immediately enhanced as soon as the interplanetary shock arrived. It has also been reported that energetic particles can be injected into the inner magnetosphere as result of these interplanetary shocks [6-8]. 
The impact of extremely powerful interplanetary shocks can result in very energetic particle acceleration within the radiation belt, which was reported on 24 March 1991 $[7,9,10]$. Ions and electrons, with energies up to $15 \mathrm{MeV}$, in the radiation belt can be accelerated by the induced electric field pulse associated with the shock. New radiation belts are then created, and last for years [11].

Although it is well known that the enhancements of energetic electron $(\mathrm{MeV})$ fluxes in the magnetosphere, including the radiation belt region, are closely related to magnetic storms [12], it remains unclear precisely how these shock-related energetic particles are produced and accelerated in the magnetosphere $[13,14]$. One type of enhancement of energetic particle fluxes $[7,9,15]$ has been found to be associated with strong SSCs rather than the main phase of a magnetic storm. This suggests that the energetic particles in the radiation belt could be produced well before the buildup of the enhanced ring current, which produces the magnetic storm. Considerable efforts have been made to understand the effects of interplanetary shocks on the population of trapped particles in the magnetosphere. Extensive studies using test particles and magnetohydrodynamic simulations have been conducted $[8,16-20]$ in order to study the particle acceleration mechanisms associated with the shock arrival. However, the particle acceleration mechanism in the radiation belt region is still not fully understood.

In the inner magnetosphere, the interaction of particles with VLF waves [21-26] and ULF waves [27-29] has been considered. Three candidate mechanisms have emerged to describe the response of particle accelerations in the radiation belt/inner magnetosphere region. They are summarized as follows:

(1) Prompt acceleration [8], for which the time scale of the acceleration process is about 1 minute. Li et al. [8] have been able to model in detail the great shock event of March 24, 1991. Friedel et al. [14] have pointed out that this model seems to be successful only for this particular shock event. The model has not proved to be applicable to other interplanetary shock impacts on the Earth's magnetosphere system. It remains unclear exactly how shock-related energetic particles are produced and accelerated within the magnetosphere $[13,14]$.

(2) Local acceleration by VLF waves, which changes the first adiabatic invariant (a few days), and diffusive radial transport, which increases energy while conserving the first adiabatic invariant. Summers et al. [30] have proposed that resonant interactions with VLF waves could heat particles with a time scale of a few days.

(3) Diffusive radial transport by ULF waves, which increases energy while conserving the first adiabatic invariant $[15,31,32]$. The time scale of this process is about tens of hours [33].

Among these candidates, VLF wave-particle interaction has been widely considered to be one of the primary electron acceleration mechanisms because electron cyclotron resonances can easily occur in the VLF frequency range. However, the time required for the VLF wave-particle interaction to accelerate electrons to relativistic energies is a few days [34], which is too long to explain the often-observed short acceleration time scales [7,35] because of the relatively small VLF wave amplitudes. ULF waves, on the other hand, can be excited with much larger amplitudes than the VLF waves in the magnetosphere by solar wind dynamic pressure variations [36,37]. ULF waves may then accelerate the energetic particles much more effectively than VLF waves. Because of the comparable periods between the drift motion of the energetic particles and the ULF oscillations, the drift-bounce resonance interaction [37-39] could be excited to adiabatically accelerate the magnetospheric particles and significantly enhance the radial diffusion coefficient [40,41]. In fact, reports have shown that there exists a close correlation between the rapid flux enhancements and ULF wave activity, for case studies $[27,42]$, as well as for statistic surveys $[33,43]$.

The solar wind and the interplanetary electromagnetic field are important energy sources for the Earth's magnetosphere. Magnetospheric ULF waves can be generated by solar wind dynamic pressure pulses. Modulations of energetic particle fluxes by waves with periods of several minutes (Pc 5 range) were first reported by Brown et al. [44]. An extensive theoretical analysis of ULF waves and particle oscillations has been carried out by Southwood and Kivelson $[37,45]$, which has been further developed to explain more observational facts $[46,47]$.

Recently, using four Cluster spacecraft observations, we have found that, subsequent to interplanetary shocks or solar wind dynamic pressure impacts on the Earth's magnetosphere, the acceleration of the energetic electrons in the radiation belt started immediately and lasted for at least a few hours. The prime acceleration mechanism was found to be the electron drift-resonance with the interplanetary shock induced ULF waves. This process is schematically shown in Figure 1.

The study of energetic electron and the ULF waves' in conjunction with interplanetary shocks is tractable from at least two obvious vantage points. One point is that, unlike other triggering mechanisms, the specific energy source is clear. The second point is that the response of the magnetospheric system to interplanetary shocks typically yields a significant and easily identifiable electromagnetic signal. Thus, there is no temporal ambiguity for interplanetary shock related phenomena.

\section{ULF Waves excited by both positive and negative solar wind dynamic pressure pulses/ shocks}

The energy coupling between the solar wind and the Earth's magnetosphere manifests itself in various ways, such as 


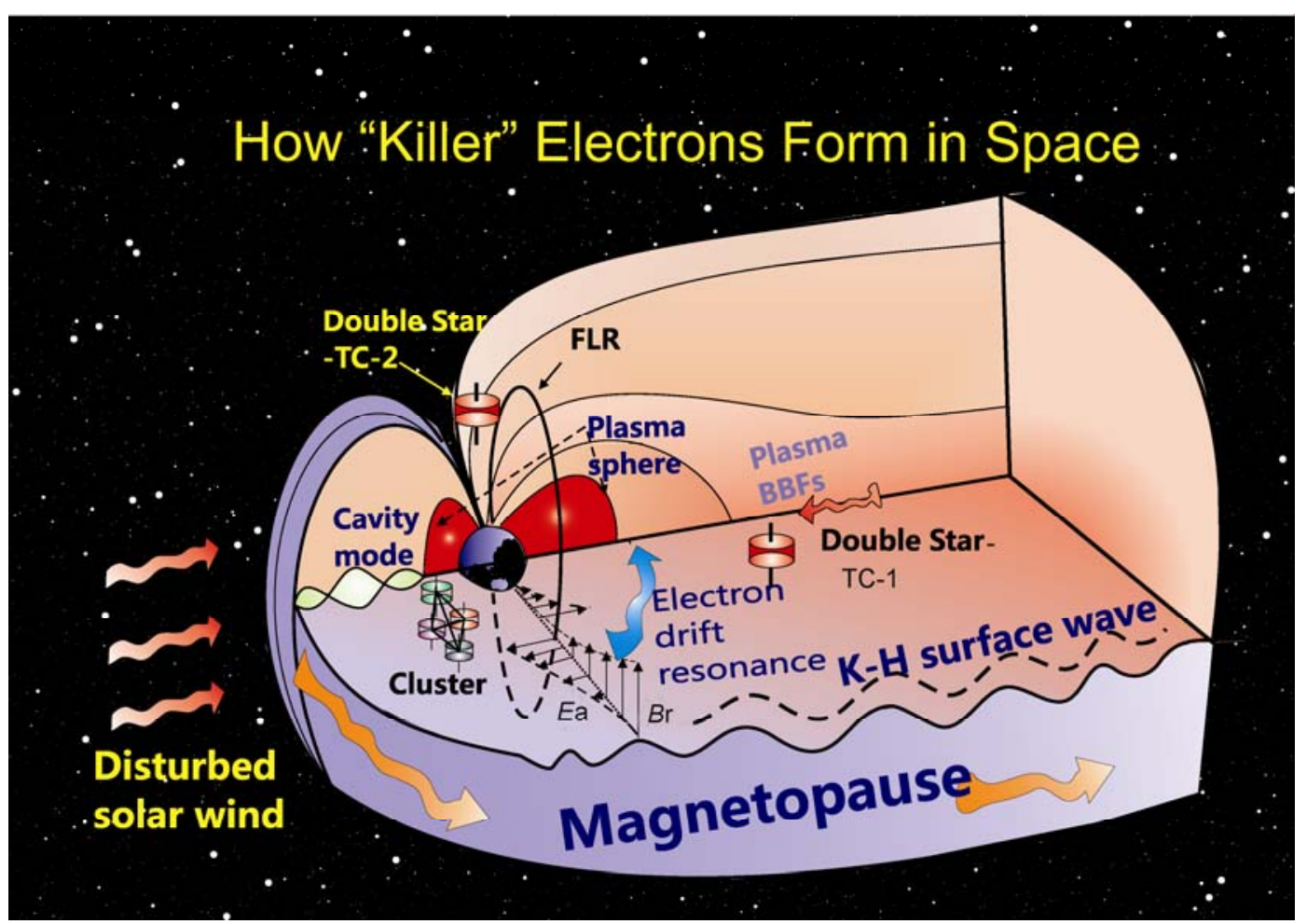

Figure 1 The wave-particle interaction between ULF waves and energetic electrons is shown schematically. Solar wind dynamic pressure impulse or Kelvin-Helmholtz surface wave stimulates the ULF waves. Field line resonances (FLR) occur under certain conditions and energetic electrons would have drift resonances with ULF waves [48].

inducing magnetic reconnection, triggering auroral activities and exciting various kinds of wave perturbations. The sudden raise/drop of solar wind dynamic pressure will cause compression/inflation of the magnetosphere [49], which further excites ULF waves in the magnetosphere [28,49].

The ULF waves in the magnetosphere are caused by either the external solar wind perturbations or the internal plasma dynamic instabilities. Kelvin-Helmholtz (K-H) instabilities and solar wind dynamic pressure pulses are generally believed to be the two most important external sources of ULF wave excitation inside the magnetosphere. The K-H instability could produce large-scale vortices, which can transport solar wind material into the magnetosphere [50,51]. Also, surface waves triggered by the K-H instability can propagate inward, via the viscous effect, exciting the FLR in the magnetosphere [36,52]. On the other hand, when a solar wind dynamic pressure pulse impinges on the magnetopause, ULF waves can be excited inside the inner magnetosphere, thus transporting solar wind energy into the Earth's magnetosphere. The above two mechanisms are unlike at the excitation of ULF wave and have different preferable occurring region (local time). The K-H instability mechanism requires a shear flow in order to satisfy the threshold condition of the instability. The main occurring region lies at the dawn and dusk flank of the magnetopause. The solar wind dynamic pressure pulses will compress the magnetopause first and then launch the fast magnetosonic wave inside the magnetosphere. This wave could further stimulate FLR in the dayside magnetosphere around the magnetic local noon [49].

The sudden raise/drop of solar wind dynamic pressure can be denoted as a positive/negative impulse. Positive impulses of solar wind dynamic pressure are often caused by the enhancement of the solar wind density, sudden change of the solar wind speed or interplanetary shock. Interplanetary shocks are often accompanied with large-scale transient solar wind phenomena such as coronal mass ejections (CMEs) or corotating interaction regions (CIRs).

The positive dynamic pressure impulses caused by interplanetary shocks associated with CMEs or CIRs are the primary cause of SSC. The magnetopause current is intensified when a positive impulse impinges on the Earth's magnetosphere. Furthermore, ULF waves are excited in the inner magnetosphere as a result of the interaction between the Earth's magnetic field and positive solar wind pressure impulses/interplanetary shocks $[28,53,54]$. Although many of the positive impulses of solar wind dynamic pressure are associated with shocks, other types of positive impulses are found to exist, e.g. the front edge of the Heliosphere Plasma Sheet (HPS) [55]. Takeuchi et al. [56] have undertaken a statistical investigation of interplanetary/solar wind sources of the solar wind dynamic negative impulses. They have found that the negative impulses are usually associated with solar wind discontinuity embedded within CIR, as well as 
the front edge of the interplanetary magnetic clouds and the trailing edge of HPS. They have also discovered, for the first time, small-scale plasma bubbles located in the CIR. Such a plasma density bubble leads to a negative impulse and a positive pressure impulse at the front and trailing edge of the plasma density bubbles. Negative and positive impulses of solar wind dynamic pressure are shown in Figure 2.

The responses of poloidal mode ULF waves to both positive and negative solar wind dynamic pressure impulses at different local time sectors are shown in Figure 3. The variations of magnetic field, $B_{\mathrm{r}}$, and electric field, $E_{\varphi}$, for poloidal waves are the result of numerical simulation. The blue solid line and red dashed line represent the poloidal ULF waves excited by positive and negative impulse respectively [49]. As shown in Figure 3, the amplitude of the poloidal wave is larger at $11 \mathrm{LT}$ than it is that at $0 \mathrm{LT}$ and 06 LT. The wave that is excited by positive and negative impulse is similar in amplitude and $180^{\circ}$ out of phase. These results have been further proved by statistical analysis of GOES magnetic field data at geosynchronous orbit [49].

\section{ULF waves interaction with energetic particles in the magnetosphere}

ULF wave is the plasma wave in the magnetosphere with frequency ranging between $1 \mathrm{mHz}$ and $1 \mathrm{~Hz}$, also known as geomagnetic pulsations. ULF waves can play an important role in mass, momentum and energy transport processes within the magnetosphere. More work is needed in order to understand the global properties of ULF waves and pre- cisely how the energy is transported from the solar wind through ULF waves to the magnetosphere, the ionosphere, and finally the ground.

It is well known that the energetic particles in the inner magnetosphere can be significantly affected by the ULF waves. The time variation scale of the Earth's magnetic field varies from a fraction of a second to tens of years. Oscillations of magnetic field lines can be sustained in the magnetospheric plasma. Lower frequency waves contain more wave power and the power levels vary roughly inversely with the wave frequency [56]. A schematic overview of the relationship between various waves and energetic electrons is shown in Figure 4. The overlap areas indicate where wave-particle resonance may occur.

Figure 4 merely gives a schematic overview. The wave frequency and wave vector, the particles' pitch angle distribution, resonance harmonic number, the location, etc. need to be taken into account so as to reveal the details of waveparticle interactions in the inner magnetosphere. Table 1 shows the different properties between toroidal and poloidal mode standing ULF waves. These differences would affect the interactions between the energetic particles and the ULF wave modes, as we shall show in section 3.

It has been suggested by, e.g. Southwood and Kivelson [45], the rate of energy change of a charged particle interacting with ULF waves (poloidal mode) in the absence of parallel electric field is

$$
\frac{\mathrm{d} W}{\mathrm{~d} t}=\mu \frac{\partial B_{\mathrm{P}}}{\partial t}+q E \cdot V_{\mathrm{d}}
$$

where $\mathrm{d} W / \mathrm{d} t, E, V_{\mathrm{d}}$, and $\mu$ are the rate of the particle energy gain, the wave-carried electric field, the particle drift veloc-

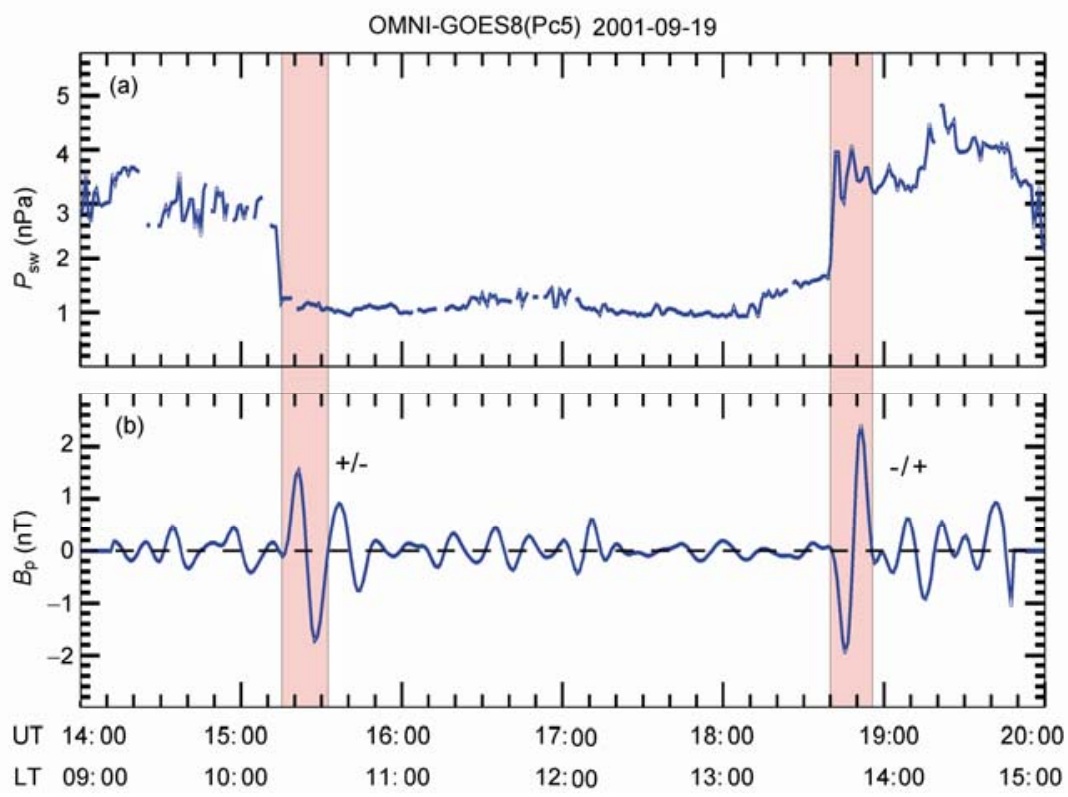

Figure 2 Negative and positive impulses of solar wind dynamic pressure (a) excited compressional ULF waves (b) at geosynchronous orbit recorded by GOES spacecraft [54]. 


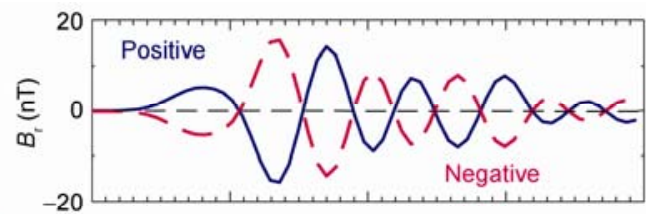

$11 \mathrm{LT}$
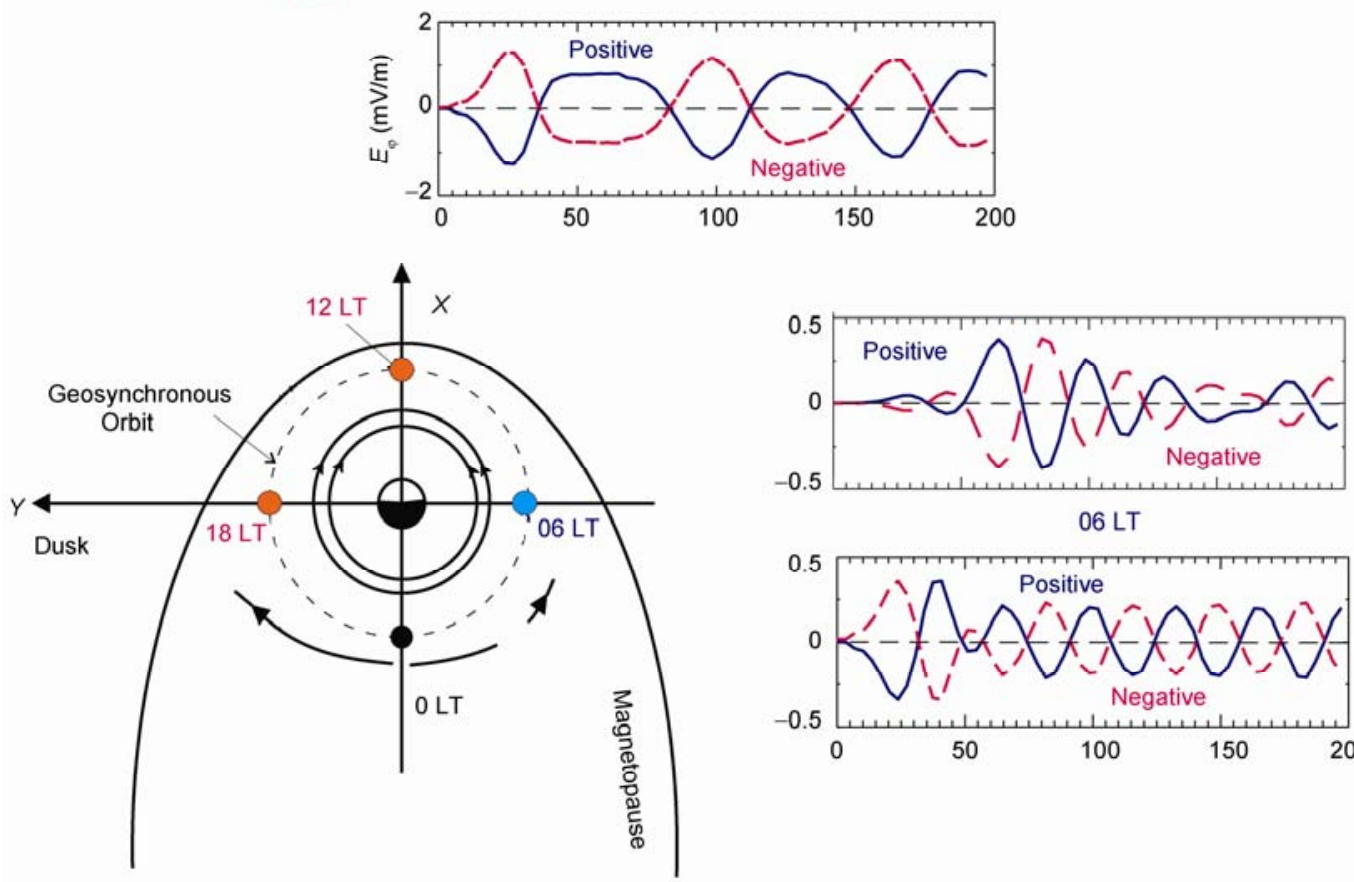

$\stackrel{E_{\infty}}{\infty}$
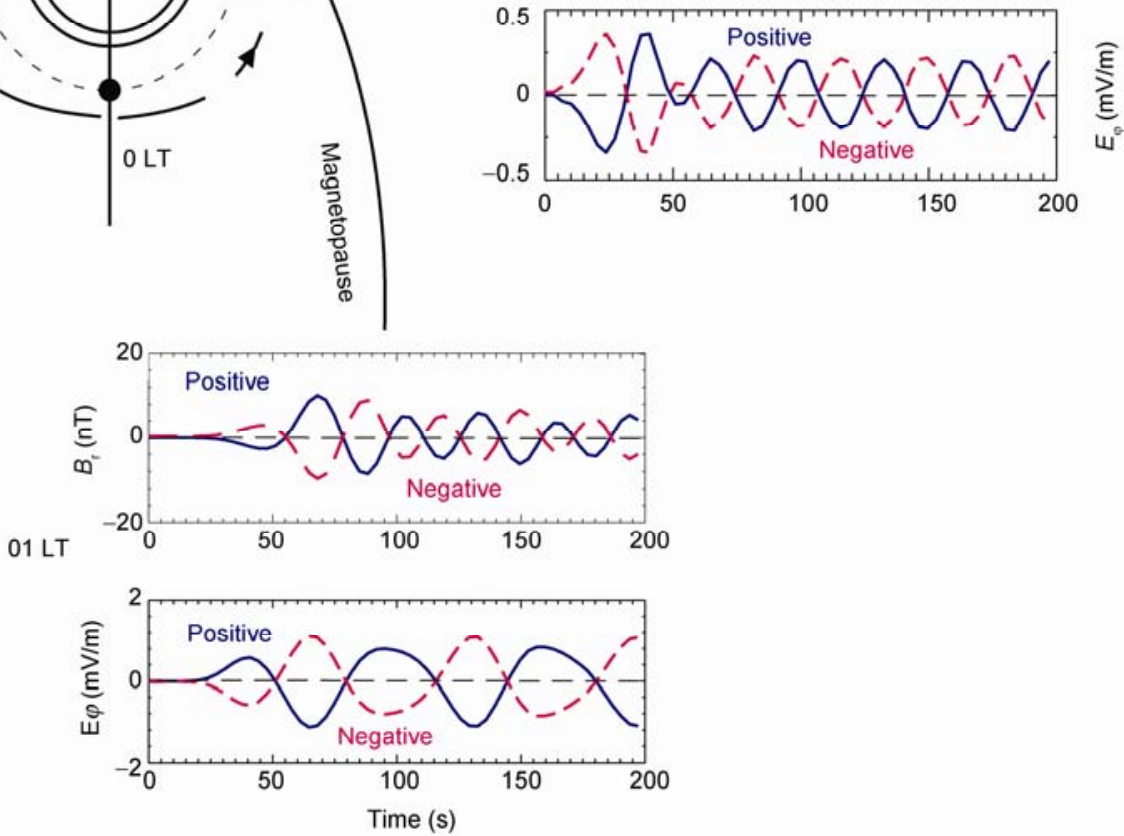

Figure 3 Illustration of the magnetic field and electric field variations of poloidal waves, excited by a solar wind positive/negative impulse, at 0,6 and 11 LT at geosynchronous orbit. The arrows schematically show the drift motion direction of ions (solid) and electrons (dashed) from midnight. The variations of magnetic field, $B_{\mathrm{r}}$, and electric field, $E_{\varphi}$, for poloidal waves are the result of numerical simulation. The blue solid line and red dashed line represent the poloidal ULF waves excited by positive and negative impulse respectively [49].

ity and the particle magnetic moment, respectively. The subscript $\mathrm{P}$ denotes the component parallel to the background magnetic field.

\section{Fast acceleration of energetic particles}

\subsection{Acceleration of energetic particles by toroidal ULF waves}

Shown in Figure 5(a) is a sketch of the electron drift path and the radial electric field orientation in a toroidal mode oscillation cycle. To reproduce global ULF toroidal waves, a snapshot of a global MHD simulation [58] is shown in Figure 5(b). The toroidal oscillation electric field vectors in the equatorial plane at 11:00 UT, Jan 10, 1997, show a low azimuthal mode number and a large scale coherence of toroidal oscillation, which is superimposed on the convection electric field pattern [58].

The basic mechanism is that electrons drift in a compressed dipole, with electric fields indicating a toroidal oscillation that is in a low mode number global toroidal wave. On the dawnside, electrons have a component of motion in 


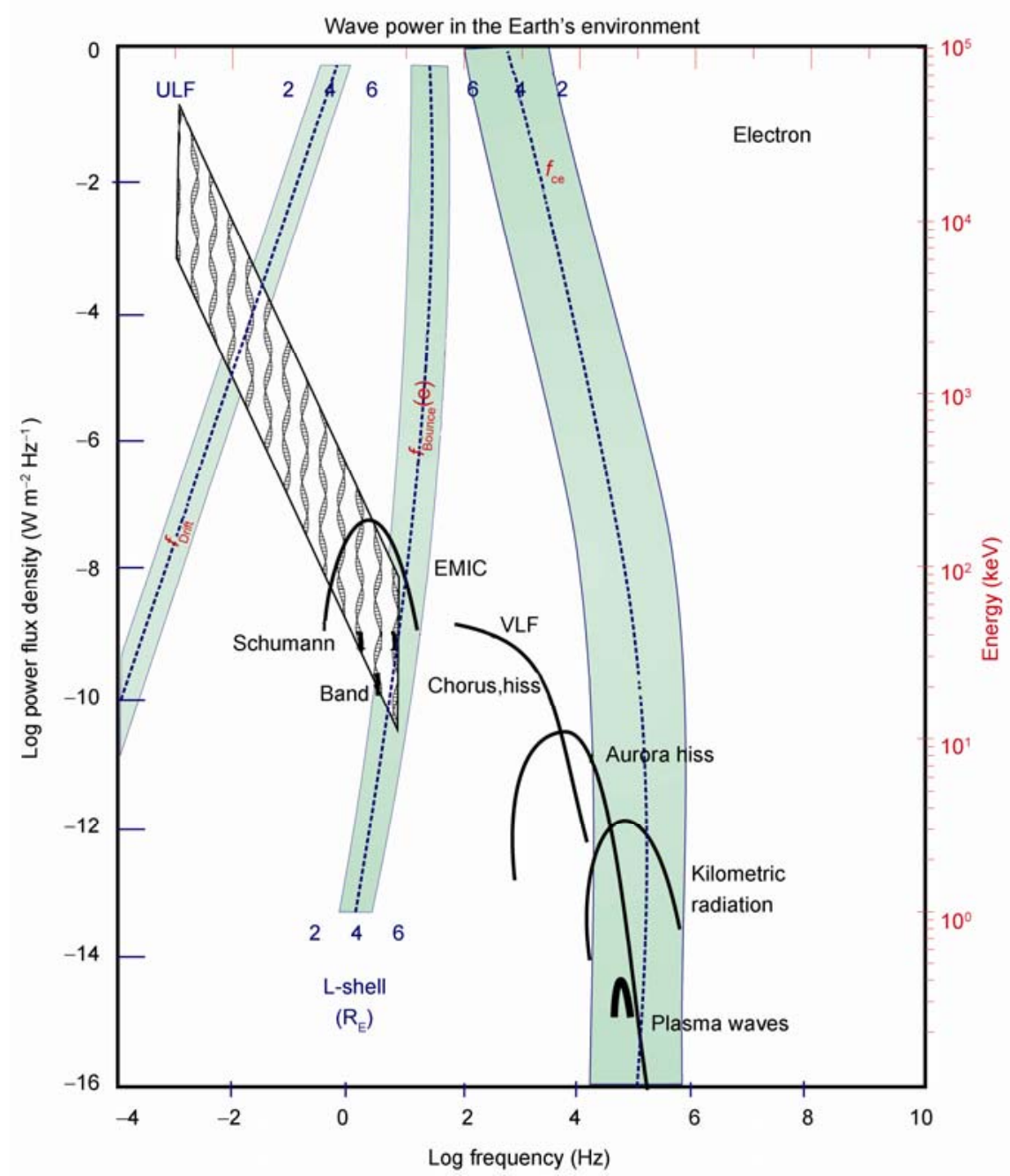

Figure 4 An overview of the possible wave-particle interactions relating to energetic electrons in the Earth's inner magnetosphere. The figure shows the power flux density of various kinds of waves with different frequency (left $Y$-axis), the gyrating bouncing and drifting frequency of energetic electrons with different energy in different L-shells (right $Y$-axis). The frequency range of ULF waves overlaps the bouncing and drifting frequency of electrons, therefore bouncing and drifting resonances may occur between ULF wave and energetic electrons [37].

Table 1 The differences between toroidal and poloidal mode standing ULF waves

\begin{tabular}{cccc}
\hline ULF mode & Magnetic Field & Electric Field & Wave number \\
\hline $\begin{array}{c}\text { Toroidal standing } \\
\text { waves }\end{array}$ & $B_{\text {azimuthal }}$ & $E_{\text {radial }}$ & Small wave number \\
\hline $\begin{array}{c}\text { Poloidal standing } \\
\text { waves }\end{array}$ & $B_{\text {radial }}$ & $E_{\text {azimuthal }}$ & Large wave number \\
\hline
\end{tabular}

the radially outward direction while the electric field points radially inward; on the duskside, a component of electron's motion and the electric field are both outward in direction. This allows for the particles to gain energy from the interaction with the ULF toroidal mode, which arises from the noon-midnight asymmetry of the geomagnetic field when the solar wind pressure is strong. As suggested by Elkington et al. [18], the resonance condition is

$$
\omega=(m \pm 1) \cdot \omega_{d} .
$$

Thus, the ULF toroidal waves are able to accelerate energetic particles in the radiation belt region. However, this mechanism requires the noon-midnight asymmetry of the geomagnetic field when the solar wind pressure is strong enough, which may not be case for the inner magnetosphere because the noon-midnight asymmetry becomes smaller and smaller as the L-shell becomes small. In a pure dipole magnetic field, this mechanism can be ignored since no noonmidnight asymmetry exists. This suggests that the acceleration of energetic electrons by the toroidal mode ULF waves becomes important in the region with a larger $\mathrm{L}$ shell (the outer magnetosphere); in smaller L shell regions (the inner magnetosphere), the poloidal mode ULF becomes responsible for the acceleration of energetic electrons.

\subsection{Fast acceleration of ions by poloidal ULF waves}

The theory of energetic particle modulation by ULF 

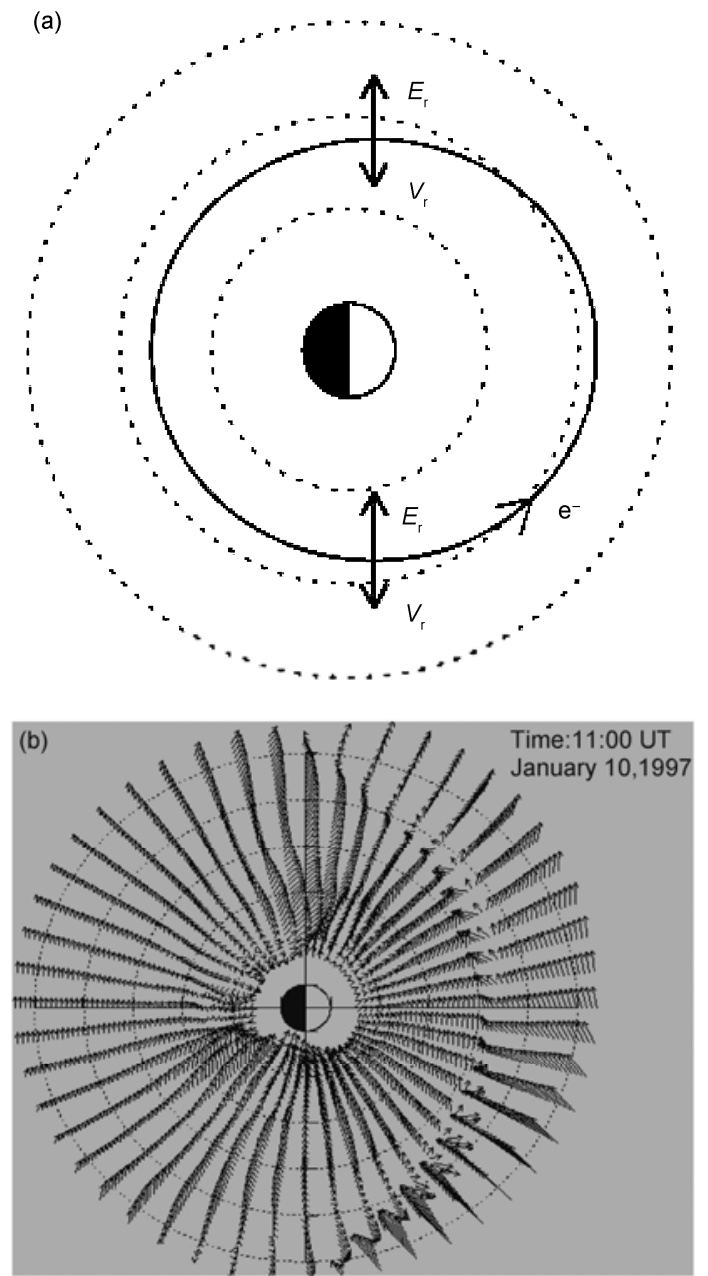

Figure 5 (a) A sketch of electron drift path and the radial electric field orientation in a toroidal mode oscillation cycle. Solid arrows indicate electric field at $t=0$ and solid line indicates the drift path of an electron with wave frequency $\omega=(m \pm 1) \omega_{d}$, the electron drift frequency, starting at dusk for an $m=2$ azimuthal mode number. The dashed lines indicate the drift path undisturbed by the ULF wave. (b) A snapshot of MHD simulation electric field vectors in the equatorial plane at 11:00 UT, showing low a azimuthal mode number and a large scale coherence of toroidal oscillation, which is superimposed on the convection electric field pattern [58].

transverse waves has been developed by Southwood and Kivelson [39,45]. In their theory, the particles experience the wave-carried electric field during their drift-bounce motions and their energy can be changed accordingly. The bounce-drift resonance condition can be determined by

$$
\omega-m \cdot \omega_{\mathrm{d}}=N \cdot \omega_{\mathrm{b}},
$$

where $N$ is an integer (normally $\pm 1, \pm 2$ or 0 ), $m$ represents the azimuthal mode number of the ULF wave, and $\omega, \omega_{\mathrm{d}}$ and $\omega_{\mathrm{b}}$ are the wave frequency, the drift frequency and bounce frequency of the particle, respectively. Because of the known energy dependence of $\omega_{\mathrm{d}}$ and $\omega_{\mathrm{b}}$, the resonance energy can be determined in theory if the wave properties are known. For the case of electrons, it should be noted that the bounce frequency is usually much higher than the other two frequencies [27,59].

Figure 6 gives a schematic view of the behavior of resonant particles in the poloidal standing waves with different harmonics. The particle behavior is examined in a stretched string model viewed in the wave frame. The westward and eastward electric fields are indicated by plus and minus signs. The magnitudes of the electric fields correspond to the density of the symbols. The blue and red dashed lines show the guiding center orbits of the resonant particles in a fundamental mode and second harmonic mode, respectively.

Considering the ions that satisfying the $N=2$ drift-bounce resonance condition in the fundamental mode, as shown in the upper part of Figure 6(a), we see that the ions will experience a strong accelerating electric field (westward) and a weak decelerating electric field (eastward) within each bounce period and thus obtain a net acceleration. In the lower part of Figure 6(a), we show the ions that satisfy the $N=1$ drift-bounce resonance condition in a second harmonic. It is apparent that they remain in the westward electric field within each bounce period and acquire successive accelerations. As for comparison, Figure 6(b) shows the guiding center orbits of the resonant particles for the situation of $N=0$ drift resonance in a fundamental and a second harmonic mode standing wave. Generally, the acceleration efficiency of ions in the second harmonic poloidal standing wave will be dominant, as compared to that in the fundamental mode.

To investigate which ring current ions can be resonant with ULF waves, we have computed $m=\left(\omega-N \cdot \omega_{b}\right) / \omega_{d}$ with $\pm 1, \pm 2$ and plotted the possible resonant energy of ring current ions (Hydrogen and Oxygen ions) versus azimuthal mode number (shown in Figure 7).

For the energy range of the ring current (10 to $500 \mathrm{keV}$ ), there are few chances to satisfy the drift-bounce resonance condition when the ULF wave azimuthal mode number are for poloidal wave $100<|m|<10$ and for toroidal wave $10<$ $|m|<1[28,60]$. As shown in Figure 7(a) and (b), the drift-bounce resonance is more important for Oxygen ions than for hydrogen ions. In the ring current energy range, oxygen ions can satisfy all $n= \pm 1, \pm 2$ conditions, which implies that the drift-bounce resonance is one of the potential mechanisms for the ring current oxygen acceleration.

Figure 7(c) and (d) shows an example of bounce-drift resonance as observed by the Cluster spacecraft. This event was found on the recovery phase of the "Halloween" storm from 21:50 to 22:20 UT, 31 Oct 2003. The Cluster spacecraft was traveling to its perigee from the southern hemisphere, near 09:00 MLT, with the magnetic L-shell changing from 9 to $6 \mathrm{R}_{\mathrm{E}}$. Panel (c) shows the $\mathrm{O}^{+}$ion spectrogram between 9 to $40 \mathrm{keV}$, where the pitch angle distribution of $\mathrm{O}^{+}$ ions with $19.4 \mathrm{keV}$ is displayed in panel (d). The pitch angle distributions exhibit clear dispersion features. The enhanced 

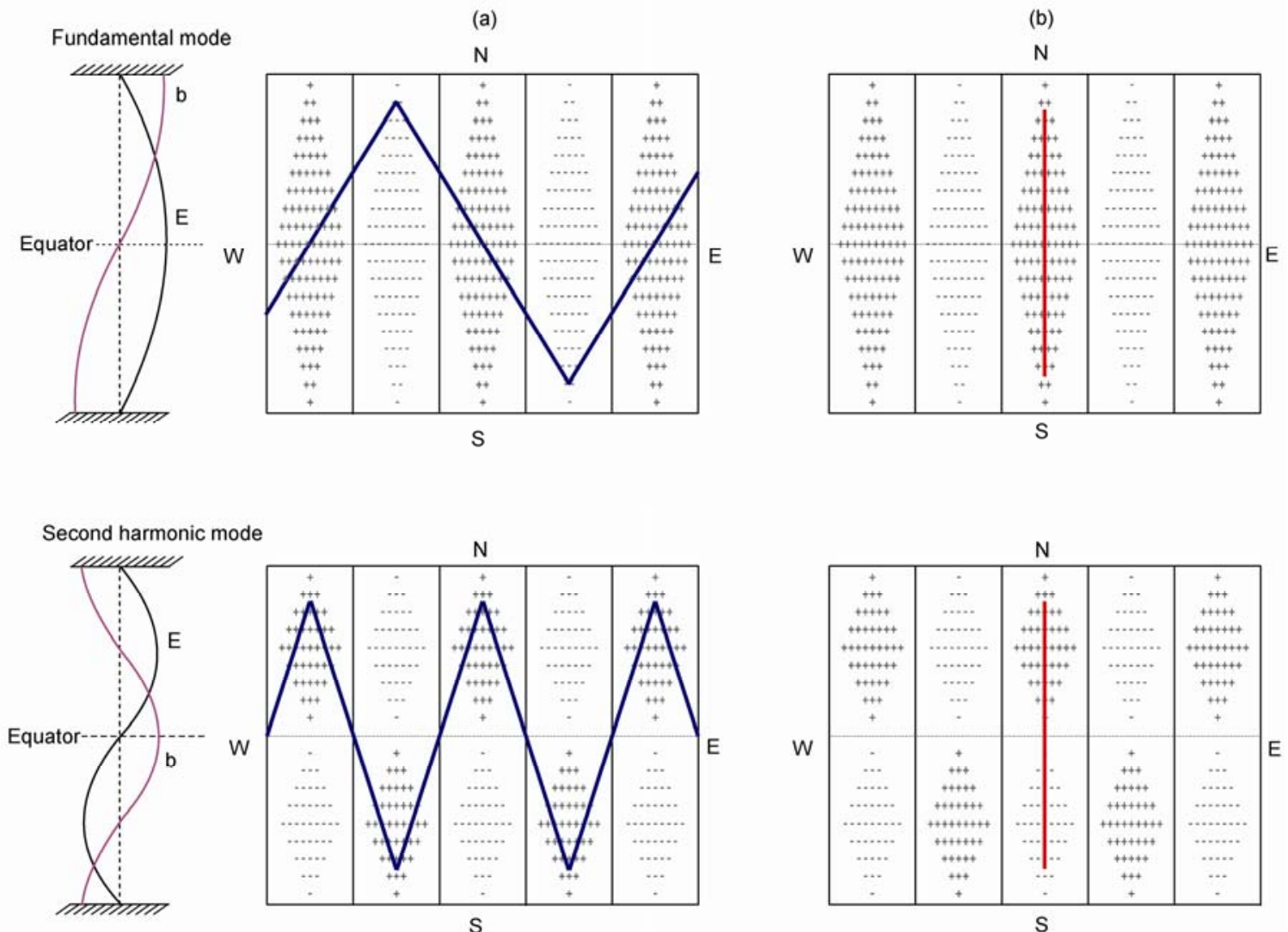

Figure 6 (a) A schematic of the behavior of resonant particle satisfying drift-bounce resonance condition $(N=2)$ in a fundamental mode standing wave as well as in a second harmonic standing wave $(N=1)$. The westward and eastward electric fields are indicated by plus and minus signs. The magnitude of the fields corresponds to the density of the symbols. (b) The same format as (a) but for the situation of drift resonance $(N=0)$ in a fundamental and a second harmonic mode standing wave.
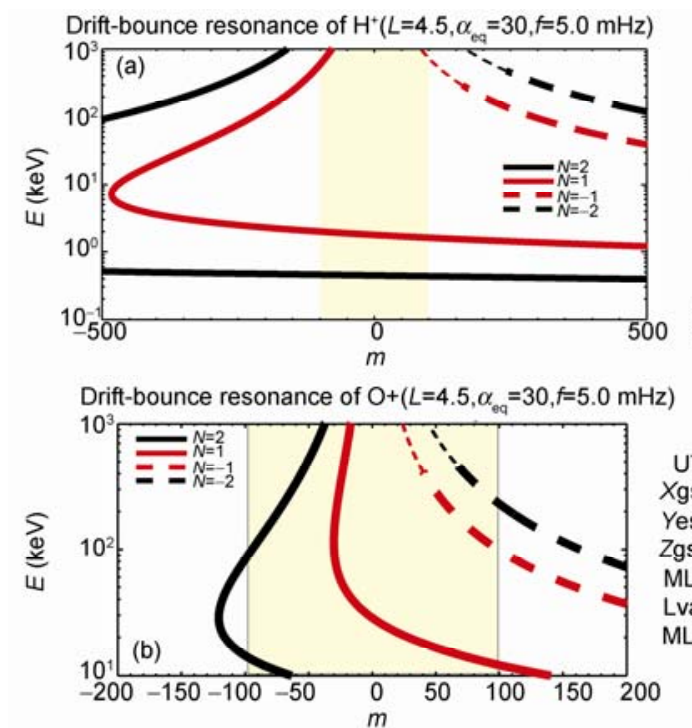
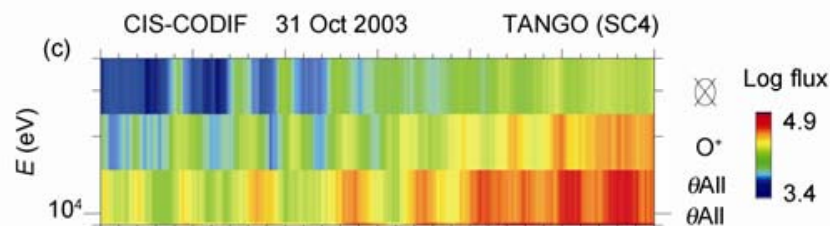

(d)
$\sigma^{150}$
ช 100

50
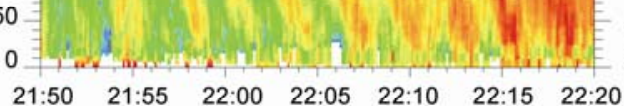

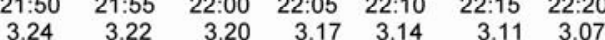

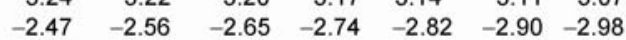

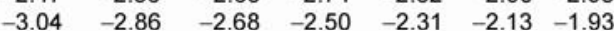

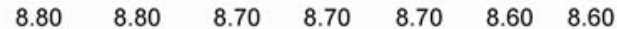

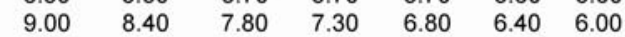

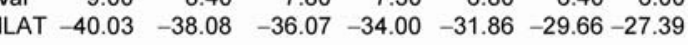


$\mathrm{O}^{+}$ions, with larger pitch angle, are observed prior to those with smaller pitch angle. The observed periodic pitch angle dispersion signatures are associated with the ULF standing waves, which have been documented in detail by Zong et al. [27].

\subsection{Fast acceleration of "killer" electrons by poloidal ULF waves}

"Killer" electrons are highly energetic particles trapped in the Earth's outer radiation belt. Their name derives from the fact that, due to their energy, they can penetrate the thick shielding of satellites and cause microscopic lightning strikes which damage and sometimes destroy vital onboard electronic components. The energy of electrons can be accelerated very efficiently when they are resonant with ULF waves. For poloidal waves, the electric field is in the azimuthal direction, which is parallel to the particle drift direction. For the energetic electrons, the electron bounce frequency is much larger than its drift frequency and the ULF wave frequency. In this situation, only the $N=0$ drift resonance can be satisfied, which implies that the electrons will not move azimuthally in the wave frame. Thus, the guiding center orbit of the electrons in resonance will appear as a vertical line, as shown in Figure 6(b). It is apparent that the acceleration and deceleration of the electron will cancel out over each bounce period in a second harmonic wave mode. Only in the fundamental mode wave could the electron experience systematic acceleration over many drift periods. Therefore, the bounce-drift resonance can only be excited at $N=0$ (Fundamental mode), which degenerates to a drift resonance with no relationship to the bounce motion

$$
\omega=m \cdot \omega_{\mathrm{d}} .
$$

The rate of energy change for the resonant particles can be written as

$$
\frac{\mathrm{d} W}{\mathrm{~d} t}=q E_{\mathrm{a}} \cdot V_{\mathrm{d}},
$$

where $\frac{\mathrm{d} W}{\mathrm{~d} t}, E_{\mathrm{a}}$, and $V_{\mathrm{d}}$ are the rate of the particle energy gain, the poloidal wave-carried azimuthal electric field, and the particle drift velocity, respectively.

A direct observation of such a scenario has been observed. As shown in Figure 8(a)-(e), during the 7 November 2004 event, the excitation of strong ULF waves was observed and found to be associated with the strong interplanetary shock encountering the magnetosphere. The fast electron acceleration can be directly correlated with the shock-induced ULF waves. With the wave-borne electric field of $40 \mathrm{mV} / \mathrm{m}$ on the drift path, a few hundreds $\mathrm{keV}$ electron can double its energy in less than a few wave periods. This is much faster than the radial diffusion time scale [61] of the ULF wave-driven diffusive process, suggesting that the observed electric field fluctuations are sufficient to explain the electron spectral increase in Figure 8(f) through drift resonance.

\subsection{Particle acceleration due to ULF waves damping}

At 18:27 UT on November 7, 2004, an interplanetary shock hit the magnetosphere. During the event, the maximum solar wind dynamic pressure reached $70 \mathrm{nPa}$. Cluster satellites in the morning side of the plasmapause $(L=4.4)$ at that time, observed ULF waves induced by the interplanetary shock impacting on the magnetosphere. The amplitude of electric field was observed to be greater than $60 \mathrm{mV} / \mathrm{m}$ [28]. Figure 9(a) shows the four Cluster satellites observations of the electric field $\mathrm{E}_{\mathrm{y}}$ during the period between 18:20 and18:40 UT.

As shown in Figure 9(b), the observed electric field (blue line) damps quickly and reaches a minimum and then gradually increases again. According to the study of Southwood [62], the main sinks of ULF wave energy include three mechanisms: damping through ionospheric Joule heating, generalized Landau damping, and mode coupling to the kinetic Alfven wave (KAW). As Southwood et al. [62] has pointed out, Joule heating in the ionosphere is generally believed to be the prime and most important damping mechanism, whereas the generalized Landau damping (which includes interactions between waves and particles with periodic motions, as well as gyration, bounce and drift energy transfer from the wave to the energetic particles) and the mode coupling mechanism are less significant. However, when the finite Larmor radius becomes important, ULF waves can couple to the KAW, while the energy in KAW can be lost in the cold plasma via Landau damping which can be very efficient. Thus, the mode coupling mechanism is significant only when the resonance width is comparable to the local ion Larmor radius, which requires that the source has an adequately narrow band and the first two damping mechanisms are inefficient. It is very interesting to examine the cases that are induced by interplanetary shocks. In the cases reported in this paper, the external source is an interplanetary shock and the shape of the solar wind dynamic pressure change is like a Heaviside step function, which implies that the source is broad band [63]. Therefore, the mode coupling mechanism is unimportant in our study. Furthermore, the ionospheric damping rate of uncoupled toroidal and poloidal standing Alfven waves can be estimated from Figure 13 of Southwood et al. [62], corresponding to our case. In our case, Cluster is in the dayside inner magnetosphere, with $L \sim 4$, and the estimated damping rate for ionospheric Joule heating is about $2 \times 10^{-4}$, which seems to be too slow for the observation results of Figure 9. On the other hand, rapid acceleration of energetic particles by ULF waves has been studied from various aspects $[27,42,64,65]$. In our observations, the rapid increase in energetic electrons and the fast wave damping rate leads us to take the generalized Landau damping as a prime energy sink that causes the fast damping of these interplanetary 

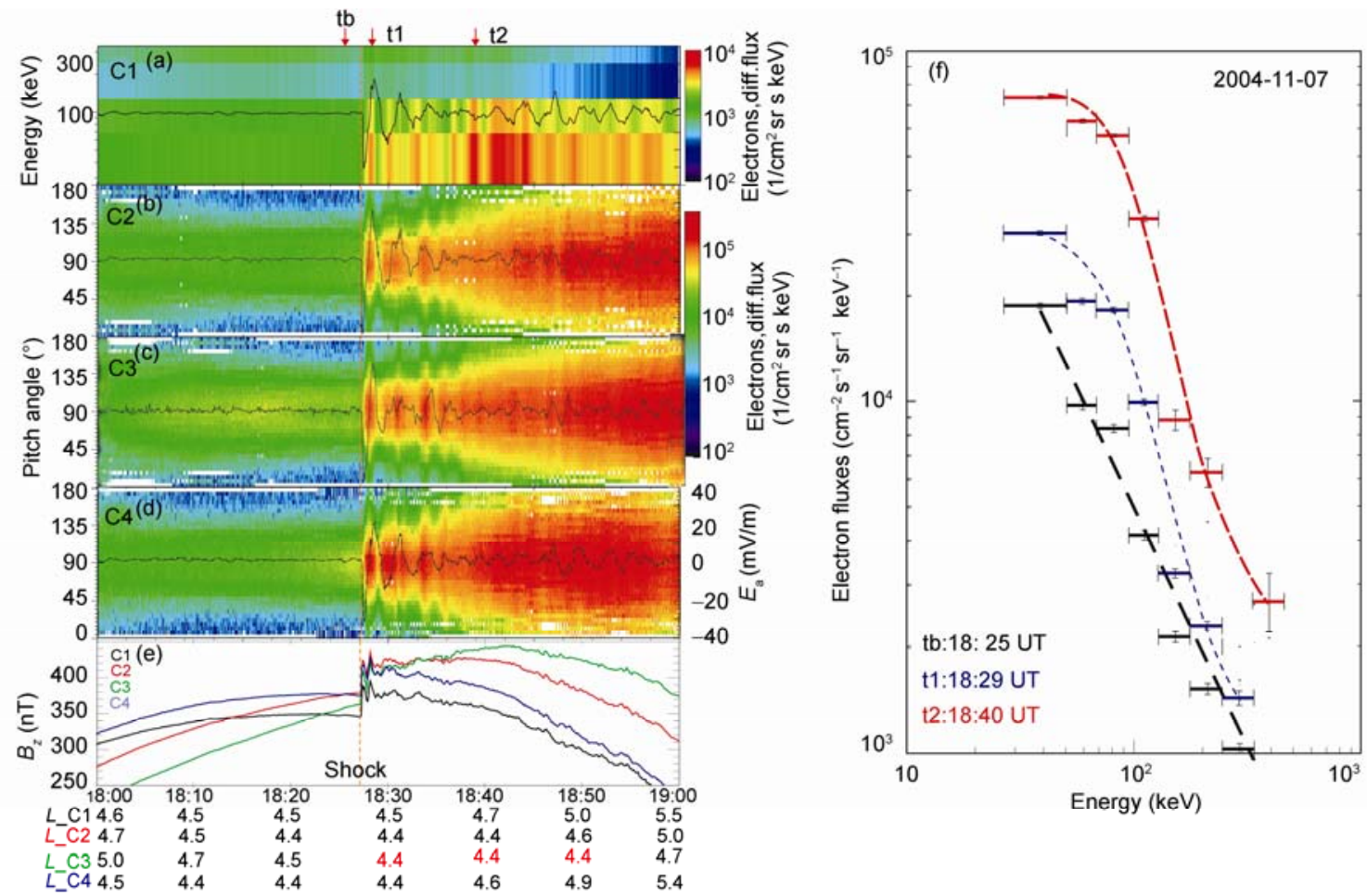

Figure 8 Panel (a)-(e) are the energetic electron spectrum measured by Cluster C1, the pitch angle distributions ( 68 to $94 \mathrm{keV}$ ) measured by C2, C3, C4 plotted along with the azimuthal electric field $E_{\mathrm{a}}$ (black line) in the mean-field-aligned (MFA) coordinate system and (e) the magnetic field $B z$ component from the four satellites in colors in the 7 November 2004 event respectively. Note that the positive $E_{\mathrm{a}}$ field is in the eastward direction. The equatorial radial distance in $\mathrm{R}_{\mathrm{E}}$, the $L$ value, for each satellite is given at the bottom. The dashed vertical line marks the time of arrival of the shock-induced field disturbances. Panel (f) shows the energetic electron spectra observed by Cluster C3 before (tb, black), immediately after (at the first peak, t1, blue), and about 12 min after the appearance of the shock induced magnetic field disturbances ( $\mathrm{t} 2$, red) in the 7 November 2004 event [28].

shock induced ULF waves. Thus, the damping of the ULF waves are separated into two terms: one term corresponds to the wave-particle interaction and energization of particles, the damping rate of this term is large and the damping is fast; the other term corresponds to the damping through the ionosphere due to its finite electric conductivity, the damping rate of this term is small and the damping is slow. We can fit the observed damping electric field to a model combining both the fast and slow damping, which results in the following formula:

$$
E(t)=A \cdot \mathrm{e}^{-D_{1} t} \sin \left(\omega_{1} t\right)+B \cdot \mathrm{e}^{-D_{2} t} \sin \left(\omega_{2} t+\phi\right),
$$

where $A=8 \mathrm{mV} / \mathrm{m}$ and $B=40 \mathrm{mV} / \mathrm{m}$ are the wave amplitudes, $D_{1}=10^{-4} \mathrm{~s}^{-1}$ and $D_{2}=2.552 \times 10^{-3} \mathrm{~s}^{-1}$ are the slow and fast damping rates, respectively, $\omega=\omega_{1}=\omega_{2}=3.984 \times 10^{-3} \mathrm{rad} \mathrm{s}^{-1}$ are the angular frequencies of the wave and $\phi=-2.66$ is the initial phase of the signal. The mean lifetime of the very large amplitude ULF wave can be obtained. For fast damping, the mean lifetime is $\tau_{1}=1 / D_{1}$. For slow damping, the mean lifetime is $\tau_{2}=1 / D_{2}$.

The red lines in Figure 9(b) are the fitted results. The slow damping rate is consistent with the estimated ionospheric damping rate as mentioned above, while the fast damping rate is at least 3-4 times larger than the slow damping rate, according to the fitted values, which may imply the rapid energy loss of ULF waves via wave-particle interactions or other processes. As pointed out by Southwood [62], the electrons are ineffective in wave damping, so it is speculated that ions may play an important role in Landau damping and cause the fast wave damping. However, the role of drift resonance with energetic electrons and drift-bounce resonance with energetic ions in wave damping needs to be investigated further through detailed theoretic analysis and numerical simulation. A detailed analysis of the wave damping mechanism is beyond the scope of this paper and would be a further topic of our study.

It is worth noting that the fast damping electric field of ULF waves implies that there exists an additional acceleration mechanism. As we have discussed previously, the drift motion of energetic electrons is mainly in the azimuthal direction pointing eastward, i.e. the direction of the poloidal wave electric field. Because the electron charge is negative, the acceleration process corresponds to the negative electric field perturbation, and the electrons are decelerated when the perturbation turns positive. However, the observed electric field oscillation is a fast damping wave, so in the second half cycle, electrons will not lose all of the energy gained in the first half cycle. This interaction over multiple cycles (a time scale of $15 \mathrm{~min}$ ) appears to be important for all 

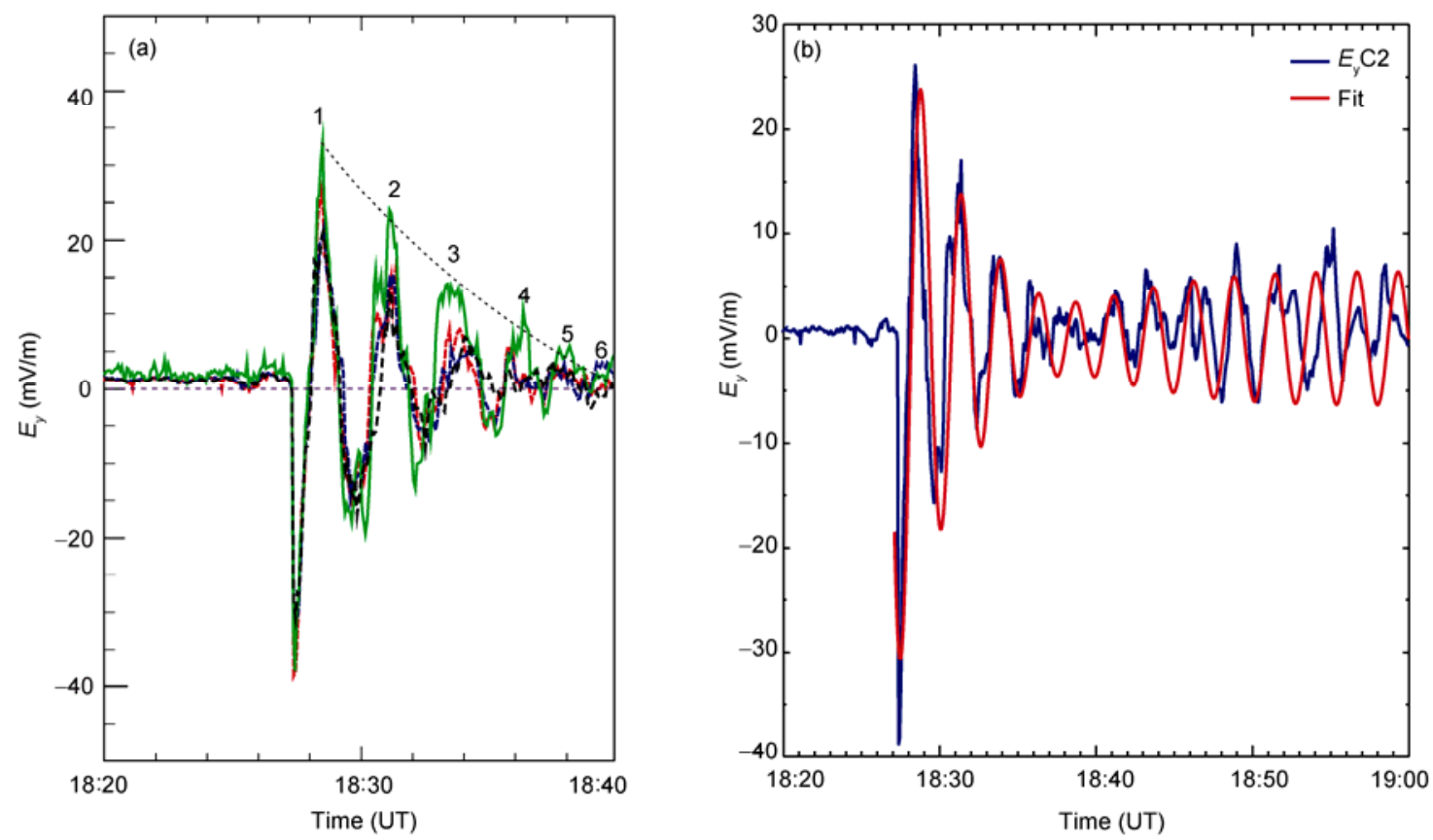

Figure 9 (a) The electric field $E_{\mathrm{y}}$ observed by Cluster C1-C4 during 18:20-18:40 UT for the 7 November 2004 event. 1-6 represent the number of peaks; (b) the observed electric field, fit to a damping model for the two cases. The $E_{y}$ electric field component observed by $\mathrm{C} 2$ (blue) and the fitted damping model value (red) for the 7 November 2004 event.

energetic particle acceleration in the inner magnetosphere. The damping ULF electric field contributes to such an acceleration process and can be estimated as

$$
\begin{gathered}
\Sigma E=\int_{0}^{\infty}\left[A \cdot \mathrm{e}^{-D_{1} t} \sin \left(\omega_{1} t\right)+B \cdot \mathrm{e}^{-D_{2} t} \sin \left(\omega_{2} t+\phi\right)\right] \mathrm{d} t, \\
\Sigma E=A \frac{\omega_{1}}{D_{1}^{2}+\omega_{1}^{2}}+B \frac{\omega_{2}}{D_{2}^{2}+\omega_{2}^{2}} .
\end{gathered}
$$

This process applies to all particles and does not require for a resonance condition.

\subsection{New radiation formation and slot region injection}

Figure 10(a) shows that a large amount of highly energetic $(2-6 \mathrm{MeV})$ electrons were injected into the slot region $2<L$ $<3.5$ on Nov. 7, 2004, immediately after an intense interplanetary shock impact on the magnetosphere. The injected electrons remained there for more than one month. As mentioned in the introduction section, there are three possible mechanisms responsible for the flux enhancement of energetic electrons during geomagnetic storms [38,66]: (1) global convective transport from the magnetotail plasma sheet, (2) inward radial diffusion driven by ULF waves and (3) local acceleration through gyro-resonant VLF waveparticle interaction. ULF wave activities are greatly enhanced globally [27,28,32] during this magnetic storm. Loto' aniu et al. [41] have calculated the rate of radial diffusion by intense ULF waves during the onset of the 29 October 2003 Halloween storm and have shown that radial diffusion can occur in the slot region near $L=2$ over a timescale of $\sim 24 \mathrm{~h}$.

To understand how the high energy electrons are injected into the slot region and the inner radiation belt in a short time scale (less than $\sim 24 \mathrm{~h}$, as shown in Figure 10(a)), equatorial electric field amplitude $\delta E_{a}$, in the slot region, and the inner magnetosphere can be obtained from measurements of the ground-based magnetic field amplitude $b_{i}$, by solving a guided poloidal Alfven mode wave equation [67], treating the wave mode as the fundamental poloidal mode for all L-shells. These results are presented in Figure 10(b)-(d). The three panels (b)-(d) show the derived electric field at $L=6.03$ (DAWS), the observed electric field by Cluster $\mathrm{C} 1$ at $L=4.4$ and the derived electric field at $L=1.17$ (EWA), respectively. These clear ULF wave features show the globally distributed ULF waves over almost all $L$-shells. ULF waves play an important role in transport energy from the solar wind to the deep magnetosphere with small $L$-shell (even for $L=1.17$ ). ULF waves carry energy and spread along the magnetic field lines and are eventually observed at geomagnetic stations on the ground, as shown in Figure 10 (b)-(d).

The electric field estimation, shown in Figure 10(b)-(d), can be used to qualitatively analyze to the possible effects of poloidal ULF waves on the behavior of energetic particles in the radiation belt region with a small $L$ shell $(L=2-4)$. With a wave-borne electric field of $40 \mathrm{mV} / \mathrm{m}$ at $L=4.4 \mathrm{R}_{\mathrm{E}}$ and $10 \mathrm{mV} / \mathrm{m}$ at $L=1.17 R_{\mathrm{E}}$, electrons with energies of a few hundreds $\mathrm{keV}$ can double their energy in less than one wave period, which is much faster than the time scale of the ULF 

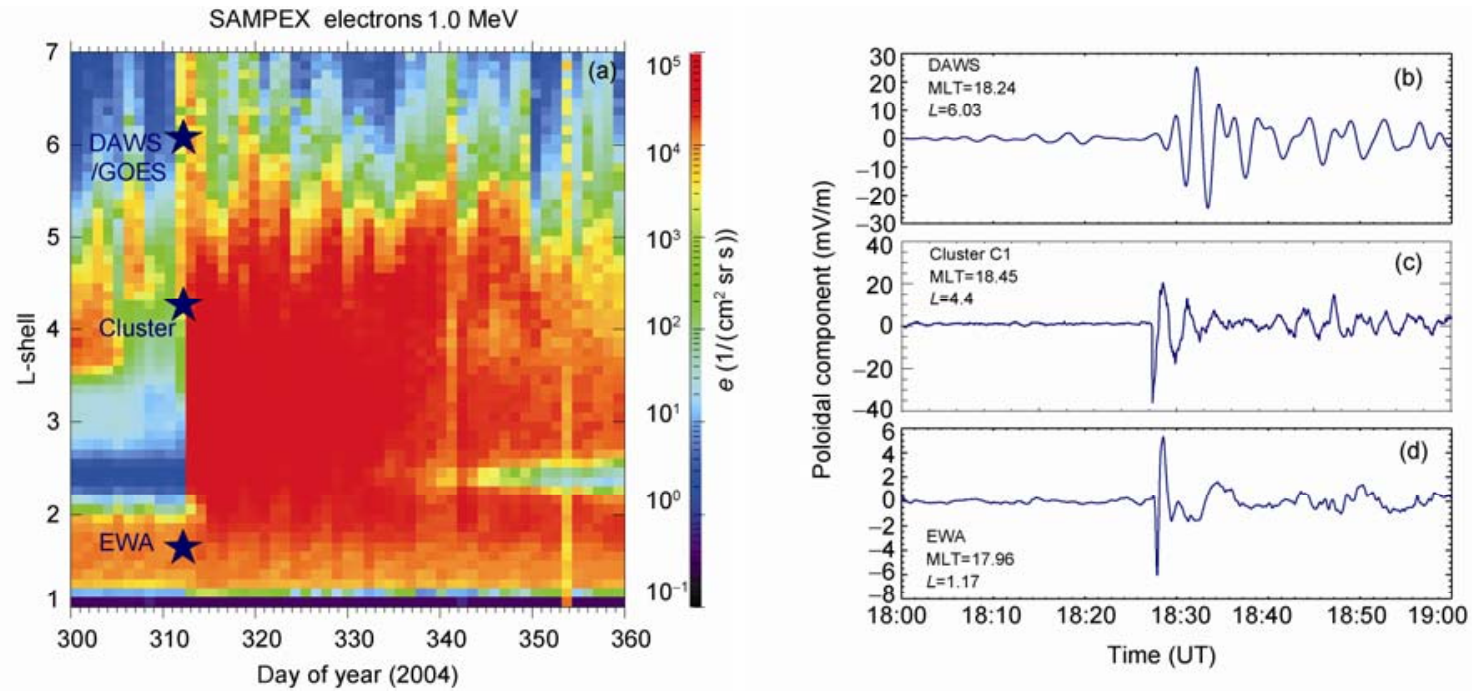

Figure 10 (a) Daily, window-averaged, color-coded on a logarithmic scale and sorted in $L$ ( $L$ bin: 0.1$)$ electron fluxes of $>1 \mathrm{MeV}\left(1 /\left(\mathrm{cm}{ }^{2} \mathrm{~s}\right.\right.$ sr)) by SAMPEX from the 300th day to the 360th day in 2004. The slot region is quickly filled in, newly injected electrons are significantly enhanced in the outer radiation belt; (b) the derived electric field for DAWS; (c) the observed electric field by Cluster C1; (d) the derived electric field for EWA. The electric fields in panels (b) and (d) are derived by treating the wave mode as the fundamental poloidal mode and solving a guided poloidal Alfven mode wave equation. See the text for details.

wave-driven diffusive processes suggested by Perry et al. [61] and Loto'aniu et al. [41]. This suggests that the observed electric field fluctuations are sufficient to explain the electron spectral increase, shown in Figure 10(a), through drift resonance acceleration, together with the damping of ULF waves due to particle acceleration.

\section{Conclusions}

The interaction between interplanetary shocks/solar wind dynamic pressure pulses and the Earth's magnetosphere manifest many important space physics phenomena, including energetic particle acceleration. In this paper we review the recent progress on the fast acceleration of "killer" electrons and energetic ions by ULF waves stimulated by the interplanetary shock in the inner magnetosphere. The objective of this paper is to understand the characteristics of the dynamic response of the Earth's magnetosphere to the impact of the interplanetary shocks and solar wind dynamic pressure pulses.

We have shown that ULF waves, excited by interplanetary shocks/solar wind dynamic pressure pulses, are generally stronger around local noon than those in the dawn and dusk flanks. We have demonstrated that disturbances induced by negative impulses are weaker than those induced by positive ones, and the poloidal wave amplitudes are stronger than the toroidal wave amplitudes, both in positive and negative events. We note that the contributions of drift-resonant acceleration by poloidal and toroidal ULF waves are different at different L-shells. The acceleration of energetic electrons by the toroidal ULF waves can be im- portant in the region with a larger L-shell (outer magnetosphere), whereas in the smaller L-shell region (inner magnetosphere), the poloidal mode ULF waves become mainly responsible for the acceleration of energetic electrons.

The enhancement of energetic electron fluxes in the radiation belt started almost immediately after the shock arrival, and the mechanism contains three contributing parts: (1) the initial acceleration due to the strong shock-related magnetic field compression; (2) the energetic particles can then be accelerated by ULF waves induced by interplanetary shocks /dynamic pressure pulses in the inner magnetosphere. This mechanism requires that energetic particles satisfy either drift-bounce resonance or drift resonance with both toroidal and poloidal waves. However, poloidal mode standing wave is much more efficient in accelerating electrons in the radiation belt region; and (3) particle acceleration due to fast damping waves. Particles will obtain a net acceleration, because particles in the second half cycle will not lose all of the energy gained in the first half cycle. This process is valid for all particles and does not require a resonance condition.

Studies on the interaction between ULF waves and energetic particles will greatly advance our understanding of the interactions of solar wind and interplanetary shocks with the Earth's magnetosphere. This is a fundamental phenomenon that occurs throughout the plasma universe, although it is uniquely accessible within the Earth's magnetosphere. This work provides fundamental information that leads to a better understanding of the process of particle acceleration by shocks, as well as the process of interplanetary shock interactions with the Earth's magnetosphere, which often cause large geomagnetic disturbances. 
This work was partly supported by the National Natural Science Foundation of China (40831061 and 41074117) and the Specialized Research Fund for State Key Laboratories.

1 Chapman S, Bartels J. Geomagnetism. Oxford: Oxford University Press, 1940

2 Brown W L, Cahill L J, Davis L R, et al. Acceleration of trapped particles during a magnetic storm on April 18, 1965. J Geophys Res, 1968, 73: 153-161

3 Matsushita S. Increase of ionization associated with geomagnetic sudden commencements. J Geophys Res, 1961, 66: 3958-3961

4 Ullaland S L, Wilhelm K, Kangas J, et al. Electron precipitation associated with a sudden commencement of a geomagnetic storm. J Atmos Terr Phys, 1970, 32: 1545

5 Ortner J, Hultqvist B, Brown R R, et al. Cosmic noise absorption accompanying geomagnetic storm sudden commencements. J Geophys Res, 1962, 67: 4169

6 Arnoldy R L, Moore T E, Akasofu S I. Plasma injection events at synchronous orbit related to positive Dst. J Geophys Res, 1982, 87: 77-84

7 Blake J B, Kolasinski W A, Fillius R W, et al. Injection of electrons and protons with energies of tens of $\mathrm{MeV}$ into L less than 3 on 24 March 1991. Geophys Res Lett, 1992, 19: 821-824

8 Li X, Roth I, Temerin M, et al. Blake, Simulation of the prompt energization and transport of radiation belt particles during the march 24, 1991 SSC. Geophys Res Lett, 1993, 20: 2423-2426

9 Vampola A L, Korth A. Eletron drift echos in the inner magnetosphere. Geophys Res Lett, 1992, 19: 625-628

10 Wygant J, Mozer F, Temerin M, et al. Large amplitude electric and magnetic field signatures in the inner magnetosphere during injection of $15 \mathrm{MeV}$ electron drift echoes. Geophys Res Lett, 1994, 21: 1739 $-1742$

11 Looper M D, Blake J B, Mewaldt R A, et al. Observations of the remnants of the ultrarelativistic electrons injected by the strong SSC of 24 march 1991. Geophys Res Lett, 1995, 22: 2079-2082

12 Reeves G D, McAdams K L, Friedel R H W, et al. Acceleration and loss of relativistic electrons during geomagnetic storms. Geophys Res Lett, 2003, 30: 1529-1533

13 Baker D N, Kanekal S G, Li X, et al. An extreme distortion of the Van Allen belt arising from the 'Halloween' solar storm in 2003. Nature, 2004, 432: 878-881

14 Friedel R H W, Reevesa G, Obara T. Relativistic electron dynamics in the inner magnetosphere-A review. J Atmos Terr Phys, 2002, 64: 265-282

15 Baker D N, Pulkkinen T I, Li X. A strong CME-related magnetic cloud interaction with the Earth's magnetosphere: ISTP observations of rapid relativistic electron acceleration on May 15, 1997. Geophys Res Lett, 1998, 25: 2975-2978

16 Hudson M K, Kotelnikov A D, Li X, et al. Simulation of proton radiation belt formation during the March 24, 1991 SSC. Geophys Res Lett, 1995, 22: 291-294

17 Elkington S R, Hudson M K, Wiltberger M J, et al. MHD/particle simulations of radiation belt dynamics. J Atmos Terr Phys, 2002, 64: $607-615$

18 Elkington S R, Wiltberger M, Chan A A, et al. Physical models of the geospace radiation environment. J Atmos Terr Phys, 2004, 66: 13711387

19 Gannon J L, Li X, Temerin M. Parametric study of shock-induced transport and energization of relativistic electrons in the magnetosphere. J Geophys Res, 2005, 110: 12206

20 Kress B T, Hudson M K, Looper M D, et al. lobal MHD test particle simulations of $>10 \mathrm{MeV}$ radiation belt electrons during storm sudden commencement. J Geophys Res, 2007, 112: 9215

21 Brice N. Fundamentals of very low frequency emission generation Mechanisms. J Geophys Res, 1964, 69: 4515-4522

22 Kennel C F, Petschek H E. Limit on stably trapped particle fluxes. J Geophys Res, 1966, 71: 1

23 Lyons L R, Thorne R M. The magnetospheric reflection of whistlers.
Planet Space Sci, 1970, 18: 1753-1767

24 Lyons L R, Thorne R M, Kennel C F. Pitch-angle diffusion of radiation belt electrons within the plasmasphere. J Geophys Res, 1972, 77 : 3455-3474

25 Abel B, Thorne R M. Electron scattering loss in Earth's inner magnetosphere 1. Dominant physical processes. J Geophys Res, 1998, 103: 2385-2396

26 Abel B, Thorne R M. Electron scattering loss in Earth's inner magnetosphere 2. Sensitivity to model parameters. J Geophys Res, 1998, 103: 2397-2408

27 Zong Q G. Ultralow frequency modulation of energetic particles in the dayside magnetosphere. Geophys Res Lett, 2007, 34: L12105

28 Zong Q G, Wang Y F, Zhou X Z, et al. Energetic electrons response to ULF waves induced by interplanetary shocks in the outer radiation belt. J Geophys Res, 2009, 114: 2009JA014393

29 Yang B, Zong Q, Wang Y F, et al. Cluster observations of simultaneous resonant interactions of ULF waves with energetic electrons and thermal ion species in the inner magnetosphere. J Geophys Res, 2010, 115: 2214

30 Summers D, Thorne R M, Xiao F. Relativistic theory of wave-particle resonant diffusion with application to electron acceleration in the magnetosphere. J Geophys Res, 1998, 103: 20487-20500

31 Rostoker G, Skone S, Baker D N. On the origin of relativistic electrons in the magnetosphere associated with some geomagnetic storms. Geophys Res Lett, 1998, 25: 3701-3704

32 Mathie R A, Mann I R. A correlation between extended intervals of ULF wave power and storm-time geosynchronous relativistic electron flux enhancements. Geophys Res Lett, 2000, 27: 3261-3264

33 O'Brien T P, Lorentzen K R, Mann I R, et al. Energization of relativistic electrons in the presence of ULF power and $\mathrm{MeV}$ microbursts: Evidence for dual ULF and VLF acceleration. J Geophys Res, 2003, 108: $1329-1342$

34 Horne R B, Thorne R M, Glauert S A, et al. Timescale for radiation belt electron acceleration by whistler mode chorus waves. J Geophys Res, 2005, 110: A03225

35 Chen Y, Reeves G D, Friedel R H W. The energization of relativistic electrons in the outer Van Allen radiation belt. Nat Phy, 2007, 3: 614-617

36 Claudepierre S G, Elkington S R, Wiltberger M. Solar wind driving of magnetospheric ULF waves: Pulsations driven by velocity shear at the magnetopause. J Geophys Res, 2008, 113: 5218

37 Zong Q G, Wang Y F, Yang B, et al. Recent progress on ULF wave and its interactions with energetic particles in the inner magnetosphere. Sci China Ser E-Tech Sci, 2008, 51: 1620-1625

38 Hudson M, Kress B, Mueller H, et al. Relationship of the Van Allen radiation belts to solar wind drivers. J Atmos Terr Phys, 2008, 70: 708-729

39 Southwood D J, Kivelson M G. Charged particle behavior in low-frequency geomagnetic pulsations: 1 . Transverse waves. J Geophys Res, 1981, 86: 5643-5655

40 Elkington S R, Hudson M K, Chan A A. Resonant acceleration and diffusion of outer zone electrons in an asymmetric geomagnetic field. J Geophys Res, 2003, 108: 111

41 Loto'aniu T M, Mann I R, Ozeke L G, et al. Radial diffusion of relativistic electrons into the radiation belt slot region during the 2003 Halloween geomagnetic storms. J Geophys Res, 2006, 111: 4218

42 Tan L C, Fung S F, Shao X. Observation of magnetospheric relativistic electrons accelerated by Pc-5 ULF waves. Geophys Res Lett, 2004, 31: 14802

43 Mathie R A, Mann I R. On the solar wind control of Pc5 ULF pulsation power at mid-latitudes: Implications for $\mathrm{MeV}$ electron acceleration in the outer radiation belt. J Geophys Res, 2001, 106: 29783

44 Brown R R, Hartz T R, Landmark B, et al. Large-scale electron bombardment of the atmosphere at the sudden commencement of a Geomagnetic Storm. J Geophys Res, 66, 1035, 1961.

45 Southwood D J, Kivelson M G. Charged particle behavior in low-frequency geomagnetic pulsations: 2. Graphical approach. J Geophys Res, 1982, 87: 1707-1710 
46 Xie L, Zhang X J, Pu Z Y, et al. Ultra low frequency waves observed by Double Star TC-1 in the plasmasphere boundary layer. Sci China Ser E-Tech Sci, 2008, 51: 1685-1694

47 Wang Y F, Fu S Y, Zong Q G, et al. Multi-spacecraft observations of ULF waves during the recovery phase of magnetic storm on October 30, 2003. Sci China Ser E-Tech Sci, 2008, 51: 1772-1785

48 Allan W, Poulter M E. ULF waves-their relationship to the structure of the Earth's magnetosphere. Reports Progr Phys, 1992, 55: 533-598

49 Zhang X Y, Zong Q G, Wang Y F, et al. ULF waves excited by negative/positive solar wind dynamic pressure impulses at geosynchronous orbit. J Geophys Res, 2010, 115: A10221

50 Fairfield D H, Otto A, Makai S, et al. Geotail observations of the Kelvin-Helmholtz instability at the Equatorial magnetotail boundary for parallel northward fields. J Geophy Res, 2000, 105: 21159-21173

51 Hasegawa H, Fujimoto M, Phan T D, et al. Transport of solar wind into Earth's magnetosphere through rolled-up Kelvin-Helmholtz vortices. Nature, 2004, 430: 755-758

52 Rae I J, Donovan E F, Mann I R, et al. Evolution and characteristics of global Pc5 ULF waves during a high solar wind speed interval. J Geophys Res, 2005, 110: A12211

53 Yang B, Fu S Y, Zong Q G, et al. Numerical study on ULF waves in a dipole field excited by sudden impulse. Sci Chin Ser E-Tech Sci, 2008, 51: 1665-1676

54 Zhang X Y, Zong Q G, Yang B, et al. Numerical simulation of magnetospheric ULF waves excited by positive and negative impulses of solar wind dynamic pressure. Sci China Ser E-Tech Sci, 2009, 52: 2886-2894

55 Winterhalter D, Smith E, Burton M, et al. The heliospheric plasma sheet. J Geophys Res, 1994, 99: 6667-6680

56 Takeuchi T, Araki A, Viljanen A, et al. Geomagnetic negative sudden impulses: Interplanetary causes and polarization distribution. J Geophys Res, 2002, 107: 1096
57 Lanzerotti L J, Southwood D J. Hydromagnetic Waves, in Solar System Plasma Physics. Volume 3. Amsterdam: North-Holland Publishing Co., 1979. 109-135

58 Hudson M K, Elkington S R, Lyon J G, et al. Radiation belt electron acceleration by ULF wave drift resonance: Simulation of 1997 and 1998 Storms. In: Song P, Singer H J, Siscoe G L, eds. Space Weather. Washington D C: AGU, 2001

59 Ozeke L G, Mann I R. Energization of radiation belt electrons by ring current ion driven ULF waves. J Geophys Res, 2008, 113: 2201

60 Baddeley L J, Yeoman T K, Wright D M, et al. Morning sector drift-bounce resonance driven ULF waves observed in artificially-induced HF radar backscatter. Ann Geophys, 2002, 20: 1487-1498

61 Perry K L, Hudson M K, Elkington S R. Incorporating spectral characteristics of Pc5 waves into three-dimensional radiation belt modeling and the diffusion of relativistic electrons. J Geophys Res, 2005, 110: 3215

62 Southwood D J, Hughes W J. Theory of hydromagnetic waves in the magnetosphere. Space Sci Rev, 1983, 35: 301-366

63 Kivelson M G, Southwood D J. Resonant ULF waves-A new interpretation. Geophys Res Lett, 1985, 12: 49-52

64 Liu W W, Rostoker G, Baker D N. Internal acceleration of relativistic electrons by large-amplitude ULF pulsations. J Geophys Res, 1999, 104: 17391-17408

65 Degeling A W, Ozeke L G, Rankin R, et al. Drift resonant generation of peaked relativistic electron distributions by Pc 5 ULF waves. J Geophys Res, 2008, 113: 2208

66 Thorne R M, Shprits Y Y, Meredith N P, et al. Refilling of the slot region between the inner and outer electron radiation belts during geomagnetic storms. J Geophys Res, 2007, 112: 6203

67 Ozeke L G, Mann I R, Rae I J. Mapping guided Alfven wave magnetic field amplitudes observed on the ground to equatorial electric field amplitudes in space. J Geophys Res, 2009, 114: 1214

Open Access This article is distributed under the terms of the Creative Commons Attribution License which permits any use, distribution, and reproduction in any medium, provided the original author(s) and source are credited. 